\title{
Effect of Normobaric and Hypobaric Hypoxia on Formant Characteristics of Human Voice
}

\author{
Savita Sondhi \\ Electrical, Electronics \& \\ Communication Engg \\ ITM University \\ Gurgaon-122017, India
}

\author{
Munna Khan \\ Electrical Engineering \\ Jamia Millia Islamia (Central \\ University) \\ New Delhi-110025, India.
}

\author{
Ritu Vijay \\ Department of Electronics \\ Banasthali University \\ Banasthali, \\ Rajasthan-304022; India
}

\author{
Ashok k. Salhan \\ Biomedical Instrumentation Division \\ Defence Institute of Physiology and Allied Sciences \\ (DIPAS), DRDO, Timarpur, \\ New Delhi-110054, India
}

\author{
S. K. Sharma \\ Biomedical Instrumentation Division \\ Defence Institute of Physiology and Allied Sciences \\ (DIPAS), DRDO, Timarpur, \\ New Delhi-110054, India
}

\begin{abstract}
Background: Hypoxia is an intensive environmental stressor which affects the psychophysiological state of an individual. It has been confirmed to influence the fundamental frequency of voice. This study aims to investigate the effect of different hypoxia conditions on formant characteristics of voice. Method: Eighteen volunteers recorded voice using a mobile phone in two phases. Study-1: Six subjects were exposed to normobaric hypoxia $(\mathrm{NH})$ for four days in a normobaric chamber. For hypobaric hypoxia same subjects were airlifted to $11500 \mathrm{ft}$ above sea level (SL) and stayed there for four days. Study-2: Out of twelve subjects, six test subjects exercised after $\mathrm{NH}$ exposure. Other six were control subjects. All 12 subjects were then airlifted to $11500 \mathrm{ft}$ and stayed at this height for six days. Obtained data was analyzed using BLISS software. Result: No change in formant's frequency was observed after $\mathrm{NH}$ exposure or at high altitude (HA). Significant increase in formant's intensity was noted after NH exposure. Formant's intensity decreased on initial exposure to HA, however it increased after acclimatization. Percentage increase in the formant's intensity after NHE without exercise was more than that with exercise. Conclusion: Hypoxic stress changes voice parameters. Formant frequency is not affected by hypoxic stress.
\end{abstract}

\section{General Terms}

Signal processing, voice analysis, human computer interface.

\section{Keywords}

Normobaric hypoxia, hypobaric hypoxia, formant frequency, formant intensity, acclimatization, high altitude.

\section{INTRODUCTION}

Mechanism of voice generation involves movement of vocal cords and muscles of larynx and articulators. These movements produce audio frequencies which constitute the basic characteristics of voice. Voice is an audible index of an individual's identity, personality and psycho-physiological state. Previous studies have shown that even stress affect the human voice besides change in the quality of inspired ambient and expired air and neuro-muscular effects on vocal cords. Stress can be due to harsh environmental conditions, physical task, cognitive load, fatigue or fear. One such environmental stressor is hypoxia. It is a condition of oxygen deficiency in the body of an individual travelling to higher altitudes [5]. At sea level, the percentage of oxygen in air is approximately $21 \%$. This percentage is maintained at high altitude (HA), though reduced atmospheric pressure leads to reduced concentration of oxygen molecules per unit volume of air reducing its availability to the body. At high altitude, changes in barometric pressure, temperature, humidity, improper food and liquid intake, affects human physiology and performance [17]. Reduced arterial $\mathrm{O}_{2}$ content and $\mathrm{O}_{2}$ delivery to the working muscles results in headache, fatigue, nausea, dizziness and sleep disorder [14]. Literature describes, that level of altitude reached, duration of hypoxic exposure and intensity of hypoxia affects the fundamental frequency $\left(\mathrm{F}_{0}\right)$, formants, and intensity of voice [2, 4, 18]. Fundamental frequency (i.e. pitch) is the frequency of vibration of vocal cord inside the larynx, whereas, formants are formed due to the vibration of air inside the vocal tract. Frequency and amplitude of formants arise from resonance in the vocal tract. While majority of studies have confirmed a significant effect of hypoxic stress on the fundamental frequency $F_{0}$ of voice $[10,13,22]$, there are very few studies which have systematically investigated the effect of acute hypoxia on the formant structure of voice. One such study, reported that there was no significant change in the formant frequencies (F1, F2, $\mathrm{F} 3$ and F4) under the influence of intensive acute hypoxia [19]. However, intensity of formants were reported to be sensitive to the level of altitude (i.e. height reached) and the duration of hypoxic exposure. Authors in [19] reported that formant intensities increased on initial exposure to $5500 \mathrm{~m}$ (18045 feet), however, on initial exposure to $6700 \mathrm{~m}$ (21982 feet), it decreased. Furthermore, prolonged stay at $6700 \mathrm{~m}$ increased the formant's intensity more than $5500 \mathrm{~m}$ [19]. So far, the effect of normobaric hypoxia followed by hypobaric hypoxia on the acoustic characteristics of same subjects have not been explored.

It is known that composition of air affects human voice. Composition of air may change in two ways. One, at high altitude, due to change in barometric pressure, humidity and temperature, the density of air decreases and by that the quantity of oxygen. Therefore, on ascending to higher altitudes, one gets less oxygen, i.e. hypoxia intensifies. Secondly, at sea level, barometric pressure remains unchanged, however, the composition of air may be changed by changing the fraction of inspired oxygen $\left(\mathrm{FiO}_{2}\right)$ via 
nitrogen dilution where nitrogen is added to ambient air to reduce $\mathrm{FiO}_{2}$. Therefore, the present study was designed to examine the effect of changes in composition of air at sea level (SL) and at high altitude (HA) on the basic characteristics of human voice. This work was a part of the larger study which was conducted to assess the effect of different hypoxic conditions on human physiology. In the present research paper, frequency-amplitude formant values of vowel $(\tilde{a})$ were examined under following 2 studies:

I. Study-1: Normobaric hypoxia and hypobaric hypoxia at $11500 \mathrm{ft}$ above mean sea level (SL).

II. Study-2: Normobaric hypoxia followed by exercise and hypobaric hypoxia at $11500 \mathrm{ft}$ above mean SL.

\section{MATERIAL AND METHODS}

Eighteen healthy male subjects (in subgroups of 6), participated in this study. All subjects were inhabitants of low altitude. For normobaric hypoxia exposure, a normobaric chamber at Biomedical Instrumentation Division, Defence Institute of Physiology and Allied Sciences (DIPAS), DRDO, New Delhi, India was used. New Delhi is at an altitude of $216 \mathrm{~m}$ (709ft) above mean sea level (SL). For this study, the normobaric chamber was simulated to an altitude of $12000 \mathrm{ft}$. Dimensions of the normobaric chamber was $4.06 \mathrm{~m} * 2.08 \mathrm{~m} *$ $2.4 \mathrm{~m}$. Temperature and humidity inside the chamber were maintained at $23^{\circ} \mathrm{C}$ and $44 \%$ respectively. For creating hypoxic environment, nitrogen was added to the ambient air inside the chamber and the percentage of oxygen was reduced to $12 \%$. Local barometric pressure at the time of study was $735 \mathrm{mmHg}$. Carbon dioxide inside the chamber was set between 0.02-0.04 percent. Concentration of oxygen and $\mathrm{CO}_{2}$ was constantly controlled with $\mathrm{CO}_{2}$ scrubber using soda lime. For hypobaric hypoxia, same subjects were airlifted to an altitude of $11500 \mathrm{ft}$ above mean sea level and stationed there for a period of 4-6 days. Cabin altitude inside the aircraft was $8000 \mathrm{ft}$ and the duration of flight was 50 minutes. No physically demanding work was given to the subjects during their stay at high altitude (HA). A medical doctor was present at all times to monitor the health of all subjects. Voice was recorded using Samsung mobile phone (S-II model). Voice database consisted of audio files in (. AMR) format with a sampling rate of $44.1 \mathrm{kHz}$ (16 bit). Audio files were converted to .wav format for further analysis. For estimation of hypoxic stress, vowel $(\widetilde{\boldsymbol{a}})$ as in DIPAS was extracted from all audio clips using PRAAT software [1]. Vocal $(\widetilde{\boldsymbol{a}})$ in DIPAS was pronounced same as $(\widetilde{\boldsymbol{a}})$ in the word 'pass'. Formant frequencies F1, F2, F3 and F4 and formant intensity of vowel $(\widetilde{\boldsymbol{a}})$ were measured using BLISS software (Freeware) developed by Brown Lab Interactive Speech System [12]. Obtained data was graphically presented using MS- Excel 2013. Statistical analysis was done by paired T- test using MS- Excel 2013.

\subsection{Study-1: Normobaric hypoxia and hypobaric hypoxia at HA}

Six healthy male soldiers (age $30 \pm 4.23$ years) participated in study-1. Participants recorded their name, employment ID no, date and place of recording. Voice was recorded outside the normobaric chamber. General information was chosen for recording in order to rule out any possibility of cognitive stress or requirement of prior training. Voice recording was done in a closed noise free room with all air-conditioners and fans switched off. After the recording, subjects were asked to sit inside the normobaric chamber for four hours. They were free to chat with each other or relax inside the chamber. After four hours, voice was recorded again inside the normobaric chamber. No recording was done in-between four hours of exposure. Same speech samples were recorded for uniformity in data. This continued for 4 consecutive days at SL. On the fifth day, all subjects were airlifted to an altitude of $11500 \mathrm{ft}$ and stayed there for four days. Five hours after arriving at $11500 \mathrm{ft}$, voice was recorded. On the following days at 11500 $\mathrm{ft}$, voice was recorded every day in the morning between 8 10am.

\subsection{Study-2: Normobaric hypoxia exposure followed by exercise and hypobaric hypoxia at HA.}

To investigate the effect of physical exercise in addition to normobaric hypoxia exposure (NHE) on human voice, a study similar to that explained in study 1 (section 2.1) was conducted. Twelve healthy male soldiers (age $29 \pm 6.75$ years) participated in this study. These subjects were different from the subjects of study-1. In this study, the subjects were divided into two groups of six each and were designated as control and hypoxia exposed test subjects. On day-1, before the start of experiment, similar introductory details (as mentioned in study-1) were recorded by all 12 subjects outside the normobaric chamber in a closed noise free room with fans and air conditioners switched off. Six test subjects were then exposed to the normobaric hypoxia for 4 hours in the chamber described in study-1. After the exposure, subjects exercised following the Queen College test protocol. They performed step up and step down exercise at the rate of 24 steps per minutes inside the chamber itself, using a low height wooden stool of 16.25 inches. Immediately after the exercise, voice of all six subjects was recorded again. On day-3, all 12 subjects travelled to $11500 \mathrm{ft}$ above mean SL by air. After five hours of reaching at this height, voice was recorded. Subjects stayed at this height for six days and recorded voice every day in the morning between 8-10am to monitor the change in their voice parameters.

\section{RESULTS}

\subsection{Results of study-1}

Effect of normobaric hypoxia $(\mathrm{NH})$ followed by hypobaric hypoxia at HA on the frequency and intensity of formants (F1, F2, F3, and F4) was investigated in this part of the study. Henceforth in this paper, SL will refer to ambient air, BE will refer to average intensity of formants of all subjects, before exposure at SL (i.e. when subjects were breathing ambient air) and $\mathrm{AE}$ will refer to average intensity of formants of all subjects after normobaric hypoxia exposure. Figure 1(a) shows the average value of formants (F1, F2, F3 and F4) of vowel $(\tilde{a})$ after normobaric hypoxia exposure (AE). Figure 1(b) and 1(c) shows the average value of formants on day-1 (i.e. initial exposure to $11500 \mathrm{ft}$ ) and on day-4 (i.e. prolonged effect of $11500 \mathrm{ft}$ ) respectively. No significant change in the frequency of formants (F1, F2, F3, and F4) was observed in all the three figures. However, significant increase $(p<0.05)$ in the intensity of formants (averaged across subjects) was observed after four days of NHE (Figure 2). On day 5, all six subjects were airlifted to $11500 \mathrm{ft}$. Initial exposure to $11500 \mathrm{ft}$ resulted in decrease $(p<0.05)$ in the intensity of formants. Subjects stayed at $11500 \mathrm{ft}$ for four days which led to increase $(p<0.05)$ in the intensity. Thus, Figure 2 indicates that normobaric hypoxia as well as hypobaric hypoxia affects the intensity of formants. 

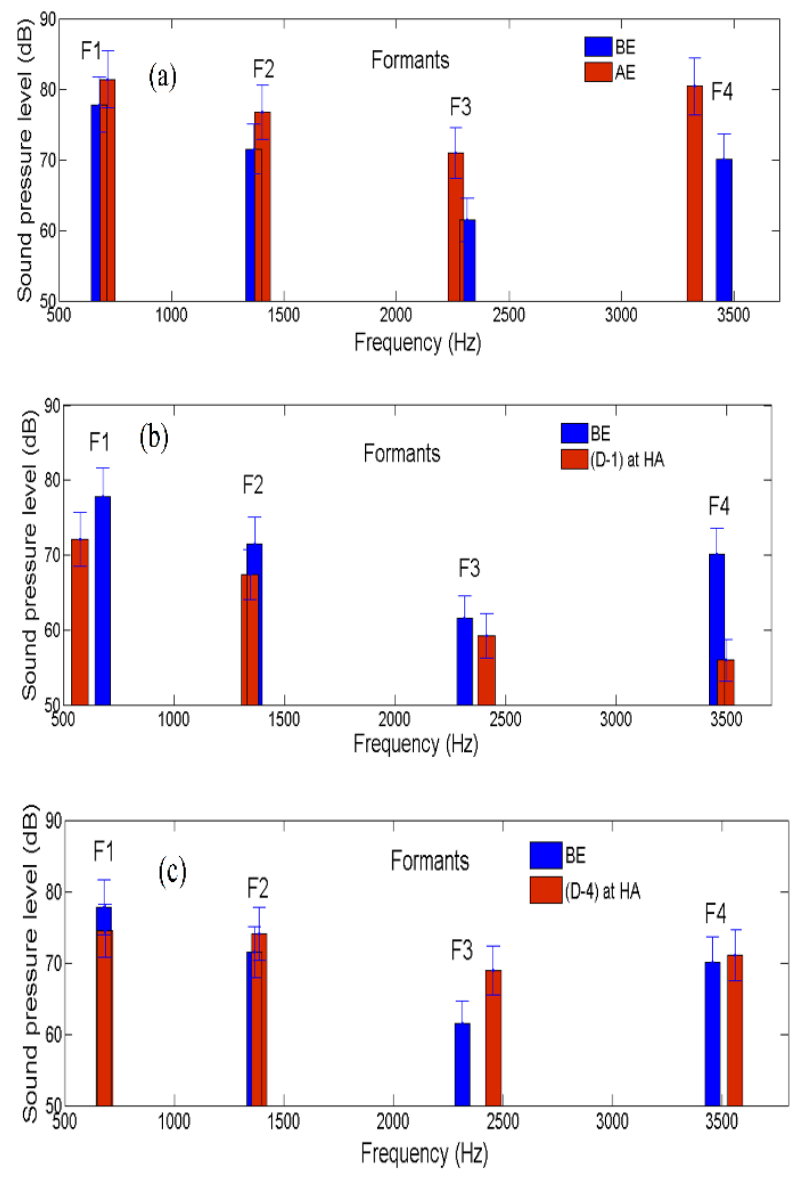

Figure 1: Average values of formants (F1, F2, F3 and F4)

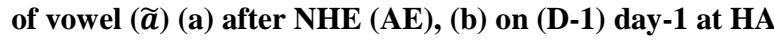
(c) on (D-4) day-4 at HA

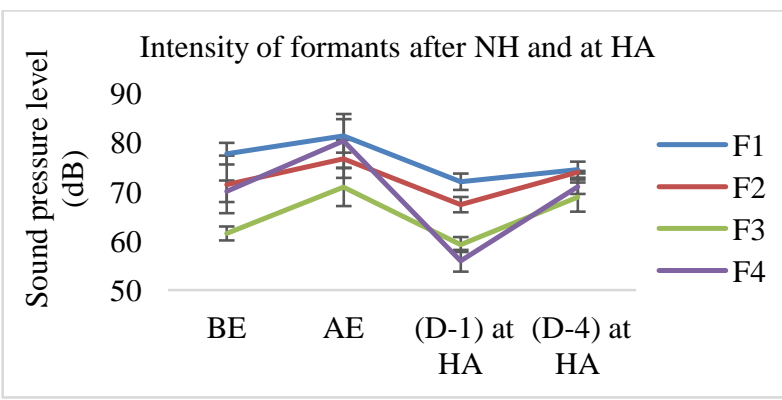

Figure 2: Shift in formant's intensity after normobaric hypoxia exposure (AE); on D-1 (day-1) at HA and on D-4 (day-4) at HA (averaged across subjects). Error bars represent standard error.

\subsection{Results of study-2}

Effect of exercise in addition to normobaric hypoxia exposure was investigated in this part of the study. The average value of formants of vowel ( $\tilde{a})$ after NHE with exercise, on day-1 (i.e. initial exposure to $11500 \mathrm{ft}$ ) and on day-6 (i.e. prolonged effect of $11500 \mathrm{ft}$ ) are shown in Figure 3(a), 1(b) and 1(c) respectively. These figures indicate no significant change in the formant frequency under the influence of normobaric hypoxia as well as hypobaric hypoxia. Figure 4(a) and 4(b) shows the shift in the intensity of formants of test group and control group respectively. Significant increase $(p<0.01)$ in formant's intensity was observed after $\mathrm{NH}$ exposure followed by exercise for test subjects (Figure 4a). Similar shift in the intensity of formants was seen in study-2 as was observed in study-1. However, on comparing the results of both these studies, it was observed that percentage increase in the intensity of formants after exercise in addition to normobaric hypoxia exposure was less (in study-2) than that observed (in study-1) without exercise. This is also depicted in Figure 7. Figure 5 compares the response of test and control subjects. Figure 5(a) displays the average value of formants (F1, F2, F3 and F4) obtained when both the groups were breathing ambient air. Both groups were then airlifted to $11500 \mathrm{ft}$ above mean sea level. Initial exposure to HA i.e. on (D-1) day-1, caused decrease in the formant's intensity $(\mathrm{p}<0.01)$ for both test as well as control group as seen in Figure 5(b). However, the percentage decrease in the intensity was more for control group than the test group (Figure 6a). Prolonged stay at HA caused increase in formant's intensity of both groups as seen on (D-6) day-6 at HA (Figure 5c). Percentage increase in intensity on day- 6 was observed to be marginally the same for both the groups (Figure 6c).
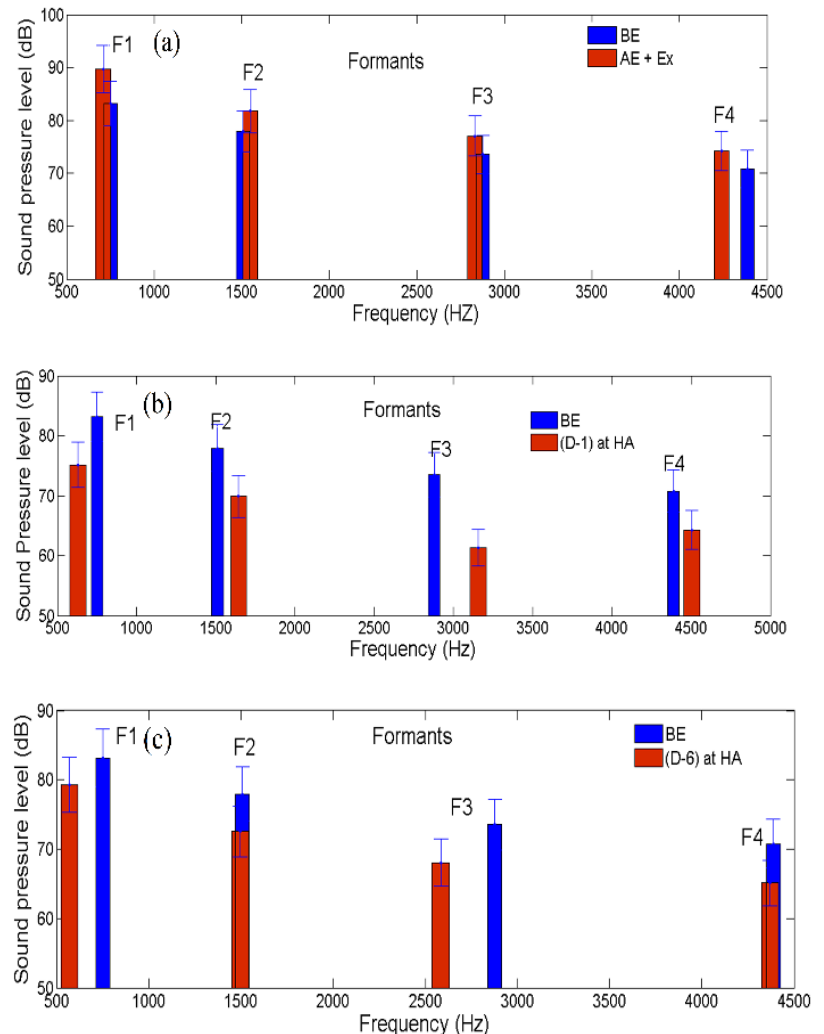

Figure. 3: Average value of formants (F1, F2, F3 and F4) of vowel ( $\widetilde{a})$ under (a) combined effect of normobaric hypoxia exposure and exercise (AE + exercise), (b) on D-1 (Day-1) at HA and (c) on D-6 (Day-6) at HA. 
(a) Test group: Intensity of formants after $\mathrm{NH}$ with exercise and at HA

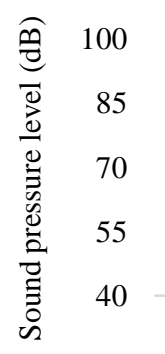

AE (D-1) at (D-6) at (b) Control group: Intensity of formant at SL and at HA

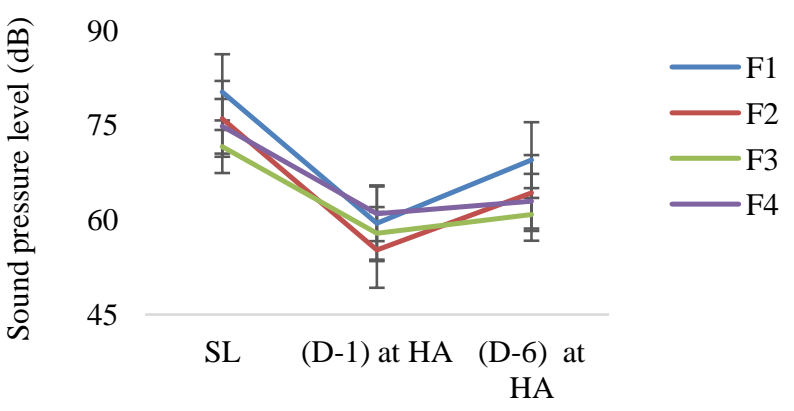

Figure 4: Shift in formant intensity of: (a) test group and (b) control group; before exposure (BE), after exposure (AE) with exercise, on (D-1) day-1 at HA and on (D-6) day-6 at HA. Error bars represent standard error.

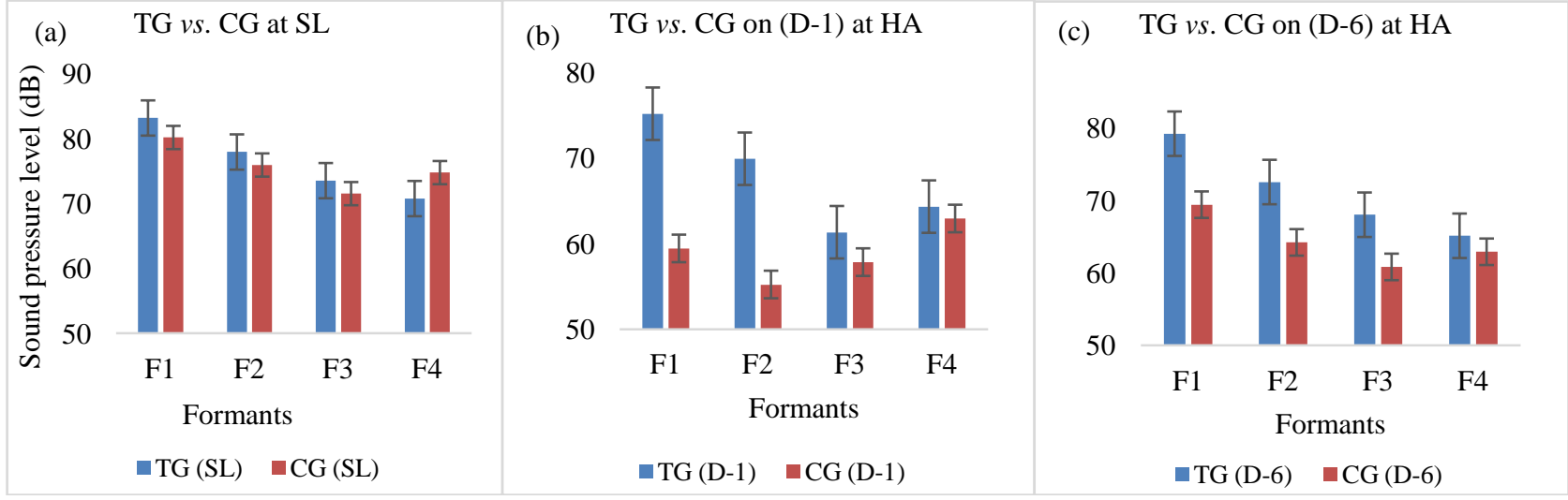

Figure. 5: Comparison of average value of formants F1, F2, F3 and F4 of test group and control group (a) at SL; (b) on (D-1) day-1 at HA and (c) on (D-6) day-6 at HA. Error bars represent standard error.

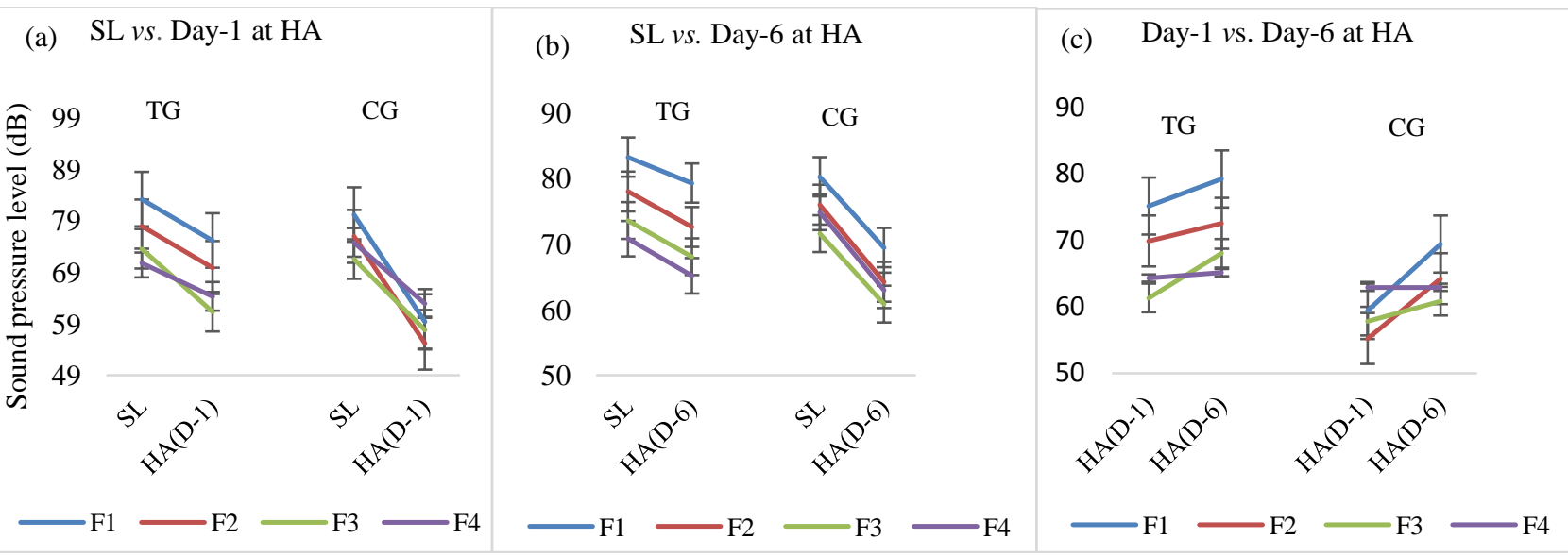

Figure. 6: Percentage change in the average of formant's intensity of test group and control group on: (a) from SL to (D-1) day-1 at HA; (b) from SL to (D-6) day-6 at HA and (c) from (D-1) day-1 to (D-6) day-6 at HA. Error bars represent standard error 


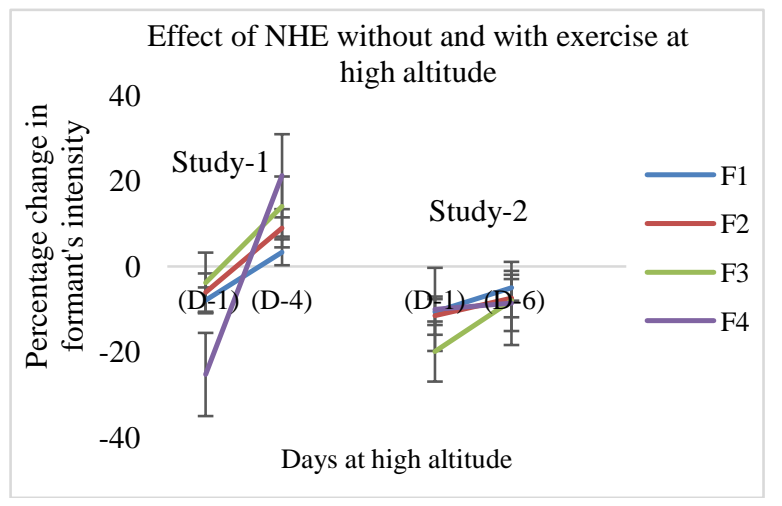

Figure 7: Comparison of effect of normobaric hypoxia exposure (NHE) with (in study-2) and without exercise (in study-1). Percentage change in formant's intensity from SL to (D-1) day-1 at HA and then from (D-1) day-1 to the last day at HA for both the studies is plotted. Error bars represent standard error

\section{DISCUSSION}

Ecological conditions of high altitude affects the laryngeal muscles. It also changes the resonant characteristics of the articulators (pharynx, nose, mouth) which affects the process of voice production. Previous studies have confirmed the influence of hypoxia on frequency, intensity and duration of $F_{0}[4,14,18]$. The present work explores the effect of different hypoxia conditions on the formant characteristics of voice.

Study-1 investigates the effect of normobaric hypoxia exposure followed by hypobaric hypoxia of $11500 \mathrm{ft}$ above sea level on the formant structure of human voice. Obtained results indicate no significant change in the formant frequency either under the influence of normobaric hypoxia or hypobaric hypoxia at high altitude (Figure 1). However, intensity of formants underwent distinct changes (Figure 2). Significant increase in the intensity of the formants was observed after normobaric hypoxia exposure. However, initial exposure to $11500 \mathrm{ft}$, on day-1 caused decrease in the intensity of formants. Normobaric hypoxia exposure in this research work was created by increasing the concentration of nitrogen and reducing the concentration of oxygen inside the normobaric chamber. As nitrogen is lighter than oxygen, the two molecular structures are different. So the quality of air had changed which may be responsible for the increase in the formant's intensity after NHE. On the other hand, on reaching high altitude, intensity of formants decreases. This may be because, at HA, air is simply rarified, its quality is same as the air at sea level. So normobaric hypoxia and hypobaric hypoxia as regards physiology may be similar but as regards to voice, changes may be there. Subjects stayed at $11500 \mathrm{ft}$ for four days. Prolonged exposure to $11500 \mathrm{ft}$ led to increase in the formant's intensity. This indicated recovery from hypoxic stress and getting acclimatized. Obtained results are in accordance with the findings of Obrenovic et al. [19] where the authors exposed one group of subjects to hypobaric hypoxia in a 'climabaro chamber' at $5500 \mathrm{~m}$ altitude and the other group to hypoxia at $6700 \mathrm{~m}$ altitude. It was reported that formants intensities increased on exposure to $5500 \mathrm{~m}$ compared to normoxia. On the other hand, initial exposure to $6700 \mathrm{~m}$ resulted in decrease in formant's intensity when compared to normoxia. However, prolonged exposure to 6700 $\mathrm{m}$ led to increase in the formant's intensity. No significant change in formant's frequency was reported in [19] due to hypoxic stress. Moreover, authors in [19] reported findings of two different groups, who were exposed to different hypoxic conditions. This paper reports the response of same subjects exposed to normobaric hypoxia followed by hypobaric hypoxia. The results of the main study indicates that exposing subjects to normobaric hypoxia prior to ascent to HA helps early acclimatization and also reduces the deleterious effects of exposure to HA.

Impact of exercise in addition to normobaric hypoxia exposure (NHE) was examined in this study. The combined effect of physical and hypoxic stress resulted in similar change in the formant's intensity and frequency as was observed in study-1. Figure 3 confirms that NHE with exercise (physical stress) does not change the formant frequency. This was in agreement with the findings of [3] where the authors analyzed formant under physical task stress and found that formants were not affected by physical stress. On the other hand, significant increase in the formant's intensity was observed after $\mathrm{NH}$ exposure followed by exercise (Figure 4a). Formant (F4) appeared to be more sensitive to NHE in both the studies as shown in Figure 2 and Figure 4a. Initial exposure to $11500 \mathrm{ft}$ (i.e. on day-1) resulted in decreases in the formant's intensity $(\mathrm{p}<0.01)$. However prolonged effect of $11500 \mathrm{ft}$ led to increase in the intensity of formants. During the stay at HA for six days, both groups behaved in the same pattern, except that the formant's intensity for test group on day-6 at HA was close to that obtained before exposure (BE) at SL. In contrast, formant's intensity of control subjects was below that measured at SL (Figure 4a and 4b). This conforms the physical acclimatization pattern of exposure to high altitude (HA). Figure 5(a-c) compares the response of both the test group as well as control group at SL and at HA. It was also noted that, percentage change in the formant's intensity for test group was less than that of control group at HA (Figure. 6(a-c)). This indicates that ecological factors of high altitude influenced the control group more than the test group. This confirms that pre-exposure to normobaric hypoxia with exercise before ascent to HA help early acclimatization and protects from acute mountain sickness (AMS).

\section{CONCLUSION}

Relationship between formant characteristics and hypoxia was observed. The following conclusions were made:

1. No significant change in the formant frequencies F1, F2, F3 and F4 was observed after normobaric hypoxia exposure or hypobaric hypoxia at HA.

2. Formant's intensity increased significantly after normobaric hypoxic exposure (NHE) both with and without exercise at sea level. Indicating that NHE had a stimulative effect on the voice production. Comparing the results of study-1 and study-2, it was also noted that percentage increase in the formant's intensity after NHE without exercise was more than that with exercise (Figure 7).

3. Initial exposure to high altitude led to decrease in the formant's intensity. However after acclimatisation, formant's intensity increased back to the range closer to normoxia.

4. Test subjects after being exposed to normobaric hypoxia before ascent to HA do not show much change in the formant's intensity on reaching HA. In contrast, 
formant's intensity significantly decreased for control group on reaching HA. This indicates that pre-exposure to $\mathrm{NH}$ prior to ascent to $\mathrm{HA}$ facilitated early acclimatization of test subject.

5. Pre-exposure to normobaric hypoxia may be beneficial for military units and mountain rescue services. This will enable them to efficiently climb to higher altitudes without supplementary oxygen and with no muscle impairment.

6. Normobaric hypoxia and hypobaric hypoxia at HA caused different effects on the intensity of formants, however the change in both hypoxic conditions was qualitatively the same.

7. The hypoxic stress affects voice parameters

\section{ACKNOWLEDGMENTS}

The work reported in this paper was conducted in the Normobaric chamber of Biomedical Instrumentation Lab, Defence Institute of Physiology and Allied Sciences (DIPAS), DRDO, Timarpur, India. Authors are very thankful to Dr. Shashi Bala Singh, Director, Defence Institute of Physiology and Allied Sciences (DIPAS), DRDO India for all the kind support and help. Gratitude is also extended to all the subjects who participated in the study.

\section{REFERENCES}

[1] Boersma P, Weenink D, PRAAT: doing phonetics by computer. (v5.3.56) 2010. Available from http://www.praat.org/ [Computer program]

[2] Davidovic J. Acoustic structure of the phoneme "I" under conditions of moderate altitude hypoxia (A 1/3 octave frequency amplitude analysis). Vojnosanit Pregl 1982; 39(6): 420-5. [Article in Serbian].

[3] Godin, K. W., and Hansen, J. H. L. (2008). "Analysis and perception of speech under physical task stress," in INTERSPEECH 2008, Brisbane, Australia, pp. 16741677.

[4] Khan M, Sondhi S, Vijay R, Sharma S, Reynolds D.B, Goswami T, Salhan A K. Effect of normobaric and hypobaric hypoxia on fundamental frequency of human voice. Aviat Space Environ Med. 2015; 86(3): 258.

[5] Liere V, Edward J, Clifford J. Hypoxia. The University of Chicago Press. Chicago and London 1963: 277-348.

[6] Lieberman P, Protopapas A, Kanki BG. Speech production and cognitive deficits on Mt. Everest. Aviat Space Environ Med.1995; 66(9): 857-864.

[7] Lieberman P. Speech physiology and acoustic phonetics: an introduction. New York: Macmillan Publishing Co; 1977.

[8] Milovanovic R, Gojkovic V. The effect of hypoxia and mental stress on the distribution of energy in the phoneme "A". Vojnosanit Pregl.1993; 50(4): 387-92. [Article in Serbian].

[9] Murray IR, Baber C, South A. Towards a definition and working model of stress and its effects on speech. Speech
Communication.1996; 20: 3-12. [Online]. Available: http:/dx.doi.org/10.1016/S0167- 6393(96)00040-4.

[10] Milkica N, Obrenovic JM, Nesic V, Cekic S, Ciric M. Intonation of pronunciation in conditions of intensive hypoxia. Acta medica Medianae. 2002; 41(7): 31-41.

[11] Mekjavic IB, Debevec T, Amon M, Keramidas ME, Kounalakis SN. Intermittent normobaric hypoxic exposures at rest: effects on performance in normoxia and hypoxia. Aviat Space Environ Med. 2012 83:942-50.

[12] Mertus J. BLISS user's manual. Providence, Brown University; 1989. http://www.cog.brown.edu/localSites/mertus/BlissHome. htm

[13] Milivojevic ZN, Milivojevic M, Brodic D, Milivojevic DR. The acute hypoxia indication by the dissonant intervals of the speech signal. International Journal of the Physical Sciences. 2012; 7(3): 394 - 399.

[14] Milivojevic ZN, Milivojevic M, Brodic D. The effects of the acute hypoxia to the fundamental frequency of the speech signal. Advances in Electrical and Computer Engineering. 2012; 12(2): 57-60.

[15] Milivojevic ZN, Brodic D, Milivojevic M. The effect of the active hypoxia to the speech signal inharmonicity. Radioengineering. 2014; 23(2): 665-670.

[16] Milivojevic ZN, Brodic D, Blagojevic D. The impact of the acute hypoxia to speech inharmonicity. Elektronika IR Electrotechnika. 2014; 20(5): 136-143. ISSN 13921215.

[17] Obrenovic J, Nesic M. Cognitive values of speech signal under the conditions of acute hypoxia. Psychology. University of Nis. 2000; 2 (2): 127-137. [Article in Serbian].

[18] Obrenovic J. Fundamental frequency of the voice in relation to hypoxia as a stressor. Facta Universitatis. Philosophy, Sociology and Psychology. 2002; 2(9): 683689.

[19] Obrenovic JM, Nesic M, Nesic V, Cekic S. Formant structure of the voice during the intensive acute hypoxia. Vojnosanit pregled. 2003; 60(2): 155-159.

[20] Pavlicek V, Schirlo C, Nebel A, Regard M, Koller EA, Brugger P. Cognitive and emotion processing at high altitude. Aviat Space Environ Med. 2005; 76 (1): 28-33.

[21] Ruiz R, Legros C, Guell A. Voice analysis to predict the psychological or physical state of a speaker. Aviat Space Environ Med. 1990; 61(3): 266-71.

[22] Saito I, Fujiwara O, Utsuki N, Mizumoto C, Arimori T. Hypoxia-induced fatal aircraft accident revealed by voice analysis. Aviat Space Environ Med. 1980; 51(4): 402-6.

[23] Smith GA. Voice analysis for the measurement of anxiety. British Journal of Medical Psychology. 1977; 50: $367-73$ 